\title{
Collet-Sicard Syndrome With Hypoglossal Nerve Schwannoma: A Case Report
}

\author{
Seung Hun Lee, MD, Eun Shin Lee, MD, Chul Ho Yoon, MD, Heesuk Shin, MD, Chang Han Lee, MD \\ Department of Rehabilitation Medicine, Gyeongsang National University Hospital, \\ Gyeongsang National University School of Medicine, Jinju, Korea
}

\begin{abstract}
Collet-Sicard syndrome is a rare syndrome that involves paralysis of 9th to 12th cranial nerves. We report an uncommon case of schwannoma of the hypoglossal nerve in a 39-year-old woman presented with slurred speech, hoarse voice, and swallowing difficulty. Physical examination revealed decreased gag reflex on the right side, decreased laryngeal elevation, tongue deviation to the right side, and weakness of right trapezius muscle. MRI revealed a mass lesion in the right parapharyngeal space below the jugular foramen. The tumor was surgically removed. It was confirmed as hypoglossal nerve schwannoma via pathologic examination. Videofluoroscopic swallowing study revealed aspiration of liquid food and severe bolus retention in the vallecula and piriform sinus. Laryngoscopy revealed right vocal cord palsy. Electrodiagnostic study revealed paralysis of the right 11th cranial nerve. In summary, we report an uncommon case of schwannoma of the hypoglossal nerve with 9th to 12th cranial nerve palsy presenting as Collet-Sicard syndrome.
\end{abstract}

Keywords Collet-Sicard Syndrome, Hypoglossal nerve, Schwannoma

\section{INTRODUCTION}

Collet-Sicard syndrome is described as unilateral paralysis of lower cranial nerves (CNs 9th to 12th). Possible causes include fractures or dissection of the base of the skull, primary or metastatic tumors, vascular disease, inflammatory disease, and iatrogenic complications [1]. Schwannoma of the hypoglossal nerve (CN 12th) is a rare and slow growing benign tumor. Most common clinical features of schwannoma of the hypoglossal nerve are uni- lateral tongue deviation, atrophy, and fasciculation [2].

In Korea, several cases of Collet-Sicard syndrome have been reported as a result of tumors, fractures, thrombosis. However, to the best of our knowledge, no case of hypoglossal nerve schwannoma causing palsy of lower cranial nerves has been reported [3-5]. Here, we report an uncommon case of schwannoma of the hypoglossal nerve with 9 th to 12 th cranial nerve palsy presenting as Collet-Sicard syndrome.

Received April 6, 2017; Accepted May 17, 2017

Corresponding author: Chang Han Lee

Department of Rehabilitation Medicine, Gyeongsang National University Hospital, 79 Gangnam-ro, Jinju 52727, Korea. Tel: +82-55-750-9639, Fax: +8255-750-8255, E-mail: ychkhk1407@gmail.com

ORCID: Seung Hun Lee (http://orcid.org/0000-0002-3596-9458); Eun Shin Lee (http://orcid.org/0000-0002-7764-1522); Chul Ho Yoon (http://orcid. org/0000-0003-4916-4180); Heesuk Shin (http://orcid.org/0000-0003-4181-2825); Chang Han Lee (http://orcid.org/0000-0001-8351-5226).

@ This is an open-access article distributed under the terms of the Creative Commons Attribution Non-Commercial License (http://creativecommons.org/ licenses/by-nc/4.0) which permits unrestricted noncommercial use, distribution, and reproduction in any medium, provided the original work is properly cited. Copyright $\odot 2017$ by Korean Academy of Rehabilitation Medicine 


\section{CASE REPORT}

A 39-year-old female patient was admitted to the Department of Otolaryngology at Gyeongsang National University Hospital due to insidious onset, hoarse voice, and unilateral wasting of tongue muscles. She complained of swallowing difficulty and discomfort in the right neck and shoulder girdle muscles. She had no history of tumor, trauma, neck mass, or meningitis. On physical examination, there was decreased gag reflex and pharyngeal sensation on the right side. Right-side tongue deviation and unilateral paralysis of the soft palate and uvula were observed. Magnetic resonance image (MRI) revealed a well-demarcated ovoid heterogeneous mass lesion in the

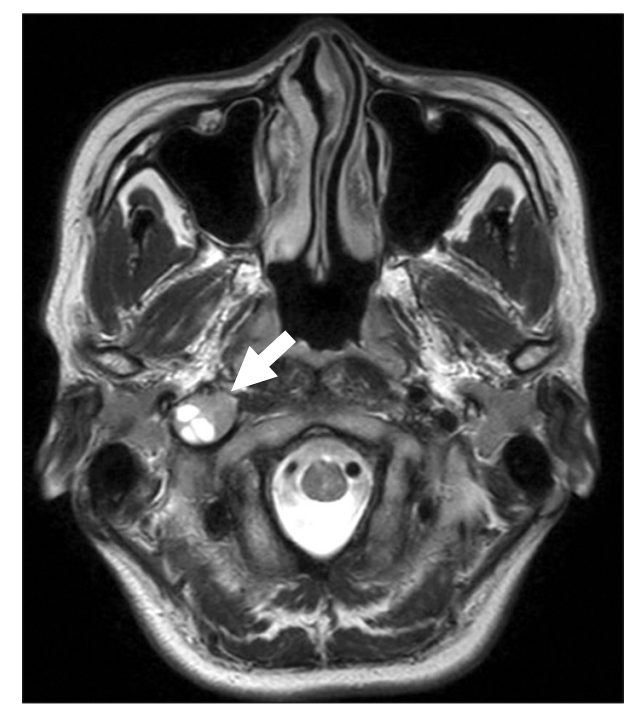

Fig. 1. Magnetic resonance image revealed a well-demarcated ovoid heterogeneous mass lesion (white arrow) in the right parapharyngeal space below the jugular foramen, measuring $2.2 \mathrm{~cm}$. right parapharyngeal space below the jugular foramen, measuring $2.2 \mathrm{~cm}$ (Fig. 1). The tumor was surgically removed. It was confirmed as hypoglossal nerve schwannoma based on pathologic examination. Post-operative complications such as edema or hematoma were not reported from enhanced CT conducted post-operatively. Four months after surgery, she was referred to the Department of Rehabilitation Medicine due to persistent swallowing difficulty and right shoulder weakness. At the time of cranial nerve examination, right-side gag reflex was reduced and right-side pharyngeal sensation decreased. Her voice was hoarse in pronunciation. Rightside deviation and atrophy of the tongue were observed (Fig. 2). Manual muscle tests revealed decreased muscle strength of Medical Research Council (MRC) grade 4 in right shoulder abduction and flexion. Laryngoscopy was

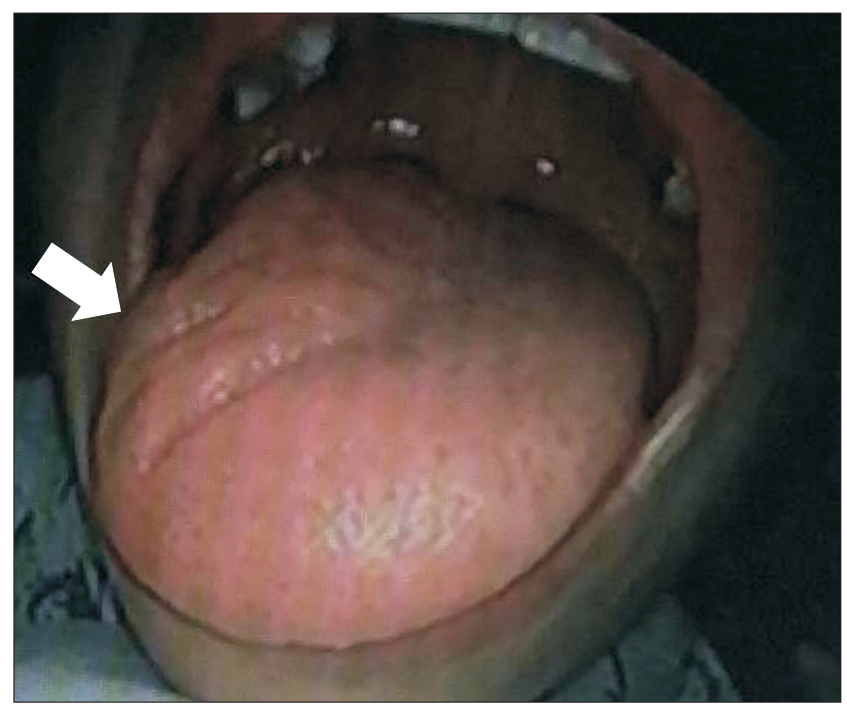

Fig. 2. The tongue deviates to the right side on protrusion (white arrow), indicating right hypoglossal nerve palsy.
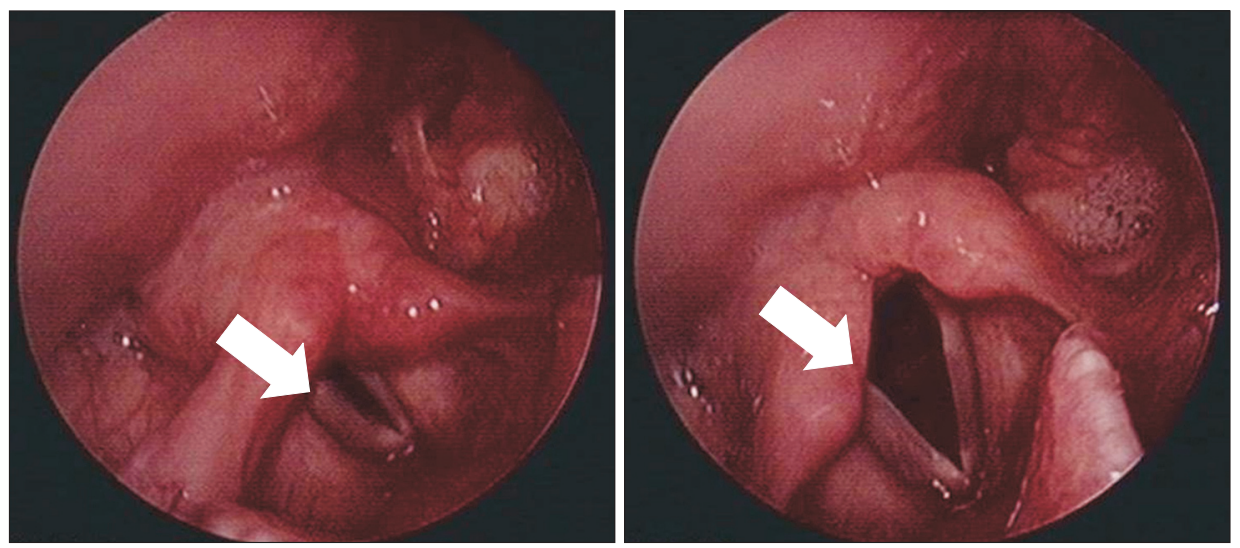

Fig. 3. Vocal cord palsy. When the patient vocalizes, only the left side of the vocal cord moves normally. The right side of the vocal cord does not move (white arrow), indicating right vagus nerve palsy. 
conducted to determine cause of hoarseness and swallowing disorder. Laryngoscopy results revealed unilateral paralysis on the right side of the soft palate and right vocal cord palsy, indicating CN 9th to 10th paralysis (Fig. 3). Videofluoroscopic swallowing study (VFSS) revealed no abnormal findings in the oral phase. However, there was decreased descent of food because of inadequate pharyngeal elevation and wall movement. Aspiration of liquid food in neutral and flexed positions and laryngeal penetration of yogurt in the neutral position were observed. Nasal regurgitation was observed in the yogurt test. VFSS revealed decreased upper esophageal sphincter opening at the beginning of the esophageal phase and severe bolus retention in the vallecula and piriform sinus (Fig. 4A, 4B). Motor nerve conduction study (NCS) revealed low amplitude of the right accessory nerve compared to that on the normal side. Needle electromyography (EMG) of right upper trapezius muscle and right sternocleidomas- toid muscle revealed abnormal spontaneous activities (Table 1). Patient's clinical findings were compatible with Collet-Sicard syndrome secondary to primary tumor on the hypoglossal nerve (CN 12th).

Patient underwent swallowing rehabilitation treatment, such as oral and pharyngolaryngeal muscle strengthening exercises, swallowing reflex therapy, and functional electrical stimulation therapy at an outpatient clinic. Follow-up VFSS at 3 months revealed laryngeal penetration and mild bolus retention in the vallecula and piriform sinus without nasal regurgitation or tracheal aspiration (Fig. 4C). Pharyngeal elevation and wall muscle movement improved. Adequate relaxation of the upper esophageal sphincter was observed. Follow-up motor NCS revealed increased amplitude of the right accessory nerve compared to the previous test. However, it did not recover to normal value. Follow-up needle EMG revealed no abnormal spontaneous activity in the right sternoclei-
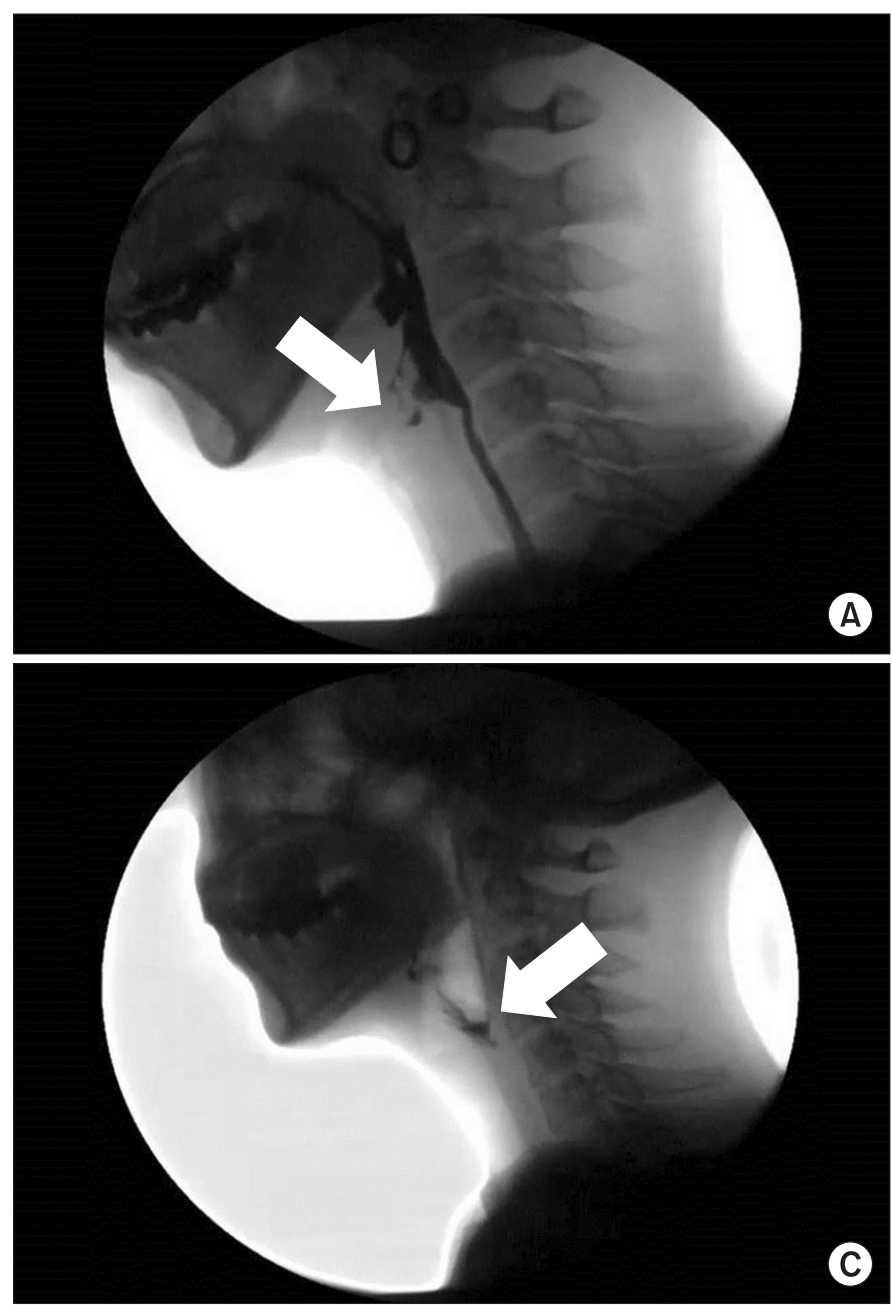

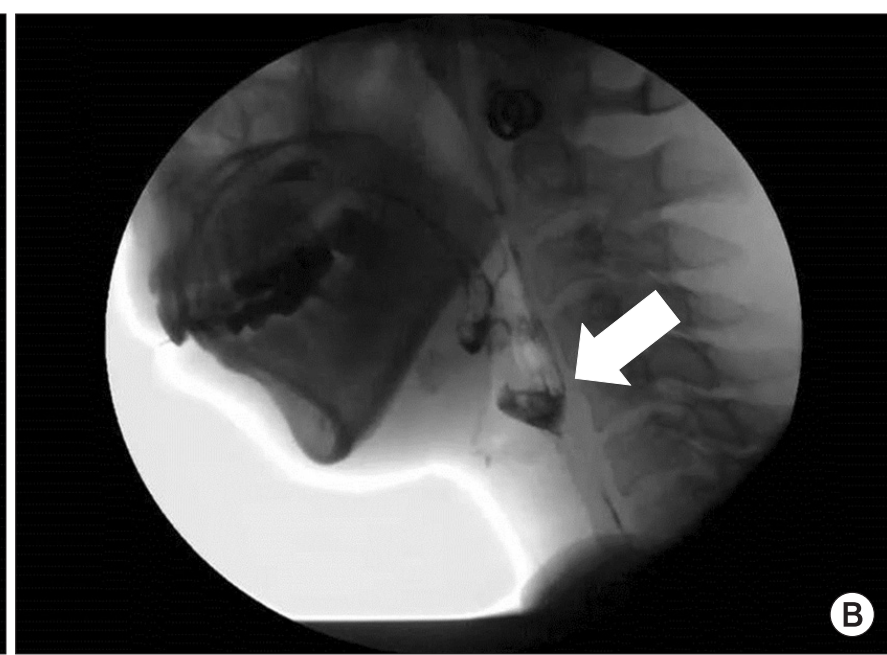

Fig. 4. Videofluoroscopic swallowing study (VFSS) shows tracheal aspiration (A) and severe bolus retention (B) in the vallecula and piriform sinus. Follow-up VFSS shows mild bolus retention (C) in the vallecula and piriform sinus. 
Table 1. Findings of needle electromyography

\begin{tabular}{|cccll|}
\hline Muscle & Fib & PSW & MUAP & Recruitment \\
\hline Initial study & & & & \\
\hline Rt sternocleidomastoid & ++ & ++ & Normal & Reduced \\
\hline Rt upper trapezius & ++ & ++ & Normal & Reduced \\
\hline Rt rhomboid major & None & None & Normal & \\
\hline Rt serratus anterior & None & None & Normal & \\
\hline Follow-up study & & & & \\
\hline Rt sternocleidomastoid & None & None & Polyphasic & Nearly full \\
\hline Rt upper trapezius & + & + & Polyphasic & Nearly full \\
\hline
\end{tabular}

Rt, right; Fib, fibrillation; PSW, positive sharp wave; MUAP, motor unit action potential.

domastoid muscle with reduced abnormal spontaneous activity in the right upper trapezius muscle. Polyphasic motor unit action potentials were observed in sternocleidomastoid and upper trapezius muscles (Table 1). Manual muscle tests revealed improved muscular strength of MRC grade 4+ in right shoulder abduction and flexion compared to previous tests. However, follow-up laryngoscopy revealed persistent right-side tongue deviation and vocal cord palsy.

\section{DISCUSSION}

Collet-Sicard syndrome involves paralysis of CNs 9th to 12th. It is characterized by loss of taste over the posterior third of the tongue (CN 9th), vocal cord paralysis and dysphasia (CN 10th), weakness of trapezius and sternocleidomastoid muscles (CN 11th), and atrophy and paresis of the tongue (CN 12th) $[2,6]$.

Schwannomas are benign tumors. They usually develop from the sensory component of cranial nerves, most commonly from CNs 8th and 10th. Hypoglossal nerve schwannoma is rare, since this nerve is purely motor [7]. CNs 9th to 11th pass through the jugular foramen, while the hypoglossal nerve (CN 12th) passes through the hypoglossal canal from the skull. As CNs 9th to 12th are near, they are often impaired at the same time. Injury to skull base and lesions involving the jugular foramen can lead to combined nerve paralysis.

Verma et al. [8] reported accompanying Collet-Sicard syndrome in a patient with jugular foramen schwannoma. Jugular foramen schwannoma arising from CNs 9th to 11th may often present as Vernet syndrome involving paralysis of CNs 9th to 11th. If paralysis of the hypoglossal nerve (CN 12th) is observed, it is diagnosed as Collet-
Sicard syndrome. However, in this case report, hypoglossal nerve schwannoma occurring from $\mathrm{CN}$ 12th caused Collet-Sicard syndrome that involves paralysis of CNs 9th to 12 th.

Garcia-Escriva et al. [9] reported a case of Collet-Sicard syndrome with schwannoma of the hypoglossal nerve. However, their diagnosis of Collet-Sicard syndrome was based on only neurological examination. Diagnosis of lower cranial nerve palsy is limited by neurological examination alone. In this case report, paralysis of glossopharyngeal and vagus nerves was confirmed by neurological examination (decreased gag reflex), clinical dysphagia symptom, laryngoscopy (vocal cord palsy), and abnormal VFSS findings. Paralysis of the spinal accessory nerve was observed in physical findings (atrophy of the trapezius muscle). It was confirmed by abnormal electrodiagnostic findings (abnormal spontaneous activity of the right upper trapezius and sternocleidomastoid muscles). Paralysis of the hypoglossal nerve was observed on neurological examination (deviation of the tongue). Our diagnosis of Collet-Sicard syndrome with hypoglossal nerve schwannoma was based on clinical findings, neurologic examination, MRI findings, VFSS, and electrodiagnostic study.

In Korea, it has been reported that Collet-Sicard syndrome can be caused by Jefferson fracture, meningioma, or jugular vein thrombosis [3-5]. Lee et al. [4] reported a case of Collet-Sicard syndrome due to cerebellopontine angle meningioma, of intracranial origin. But in this case report, hypoglossal nerve schwannoma was in the parapharyngeal space, and was of extracranial origin. According to Hoshi et al. [10], extracranial hypoglossal schwannomas are extremely rare. We report the first case of Collet-Sicard syndrome with hypoglossal nerve schwannoma in Korea, due to an uncommon cause. 
After rehabilitation treatment, patient's swallowing difficulty and right shoulder weakness improved. Therefore, careful neurological examination should be conducted to determine symptoms or signs of cranial nerve palsy after diagnosis of lower cranial nerve tumor. In addition, it is crucial to conduct electrodiagnostic study and VFSS for establishing diagnosis of lower cranial nerve palsy and provide rapid rehabilitation treatment for neurological improvement.

\section{CONFLICT OF INTEREST}

No potential conflict of interest relevant to this article was reported.

\section{REFERENCES}

1. Gutierrez Rios R, Castrillo Sanz A, Gil Polo C, Zamora Garcia MI, Morollon Sanchez-Mateos N, Mendoza Rodriguez A. Collet-Sicard syndrome. Neurologia 2015;30(2):130-2.

2. Baghel PS, Gupta A, Tripathi VD, Reddy DS. Hypoglossal schwannoma presenting as hemi-atrophy of the tongue. Neurol India 2013;61:324-5.

3. Moon TS, Sung KB, Shin DJ. A case of Collet-Sicard syndrome resulting from jugular vein thrombosis. J
Korean Neurol Assoc 1994;12:348-53.

4. Lee SI, Yang HS, Chun MH. Collet-Sicard syndrome with dysphagia due to cerebellopontine angle meningioma: a case report. J Korean Acad Rehabil Med 2006;30:402-5.

5. Kwon HC, Cho DK, Jang YY, Lee SJ, Hyun JK, Kim TU. Collet-Sicard syndrome in a patient with jefferson fracture. Ann Rehabil Med 2011;35:934-8.

6. Caldemeyer KS, Mathews VP, Azzarelli B, Smith RR. The jugular foramen: a review of anatomy, masses, and imaging characteristics. Radiographics 1997;17: 1123-39.

7. Kawakami R, Kaneko T, Kadoya M, Matsushita T, Fujinaga $\mathrm{Y}$, Oguchi $\mathrm{K}$, et al. Schwannoma in the sublingual space. Dentomaxillofac Radiol 2004;33:259-61.

8. Verma V, Singh MP, Gupta AK, Singh TP, MM A, Jaiswal N. Jugular foramen schwannoma presenting as Collet-Sicard syndrome: a rare entity. J Indian Acad Clin Med 2011;12:227-9.

9. Garcia-Escriva A, Pampliega Perez A, Martin-Estefania C, Botella C. Schwannoma of the hypoglossal nerve presenting as a syndrome of Collet-Sicard. Neurologia 2005;20:311-3.

10. Hoshi M, Yoshida K, Ogawa K, Kawase T. Hypoglossal neurinoma: two case reports. Neurol Med Chir (Tokyo) 2000;40:489-93. 